\title{
The 'lexicon of love': Understanding types of relationships as primary contexts of STI transmission
}

\author{
Pothoulaki, M. ${ }^{1}$, Vojt, G. ${ }^{1}$, Mapp, F. ${ }^{2}$, Woode Owusu, M. ${ }^{2}$, Cassell, J. ${ }^{3}$, Mercer, C. ${ }^{2}$, Nandwani, R. ${ }^{4}$, \\ Saunders, J. ${ }^{2}$, Symonds, M. ${ }^{5}$, Wayal, S. ${ }^{2}$, Flowers, P. ${ }^{1}$ \& Estcourt, C. ${ }^{1}$
}

${ }^{1}$ Glasgow Caledonian University, ${ }^{2}$ University College London, ${ }^{3}$ Brighton and Sussex Medical School, ${ }^{4}$ NHS Greater Glasgow and Clyde, ${ }^{5}$ Barts Health NHS Trust

\begin{abstract}
Introduction
STI transmission is socially patterned across different types of relationships (Mercer et al., 2016), social and sexual networks, communities and other population groups (Adimora \& Schoenbach, 2005; Harling et al., 2013). It is important to understand the ways these social contexts can enable improved targeting and tailoring of service delivery and specific sexual health interventions. Although tailoring services to people on the basis of their gender or sexual identity is established, to date, little attention has been given to how we might target service provision according to the type of relationship within which STIs are transmitted. In order to develop these ideas we need a greater understanding of relationship contexts and partner types. Here we report our analysis of qualitative data collected from the public through exploratory focus groups.
\end{abstract}

\begin{abstract}
Methods
Six focus groups with participants from the public took place in Glasgow and London. Purposive and convenience sampling was used to recruit young heterosexual participants (18-30 years) and men who have sex with men (18-65 years). Participants received a $£ 30$ shopping voucher. Each focus group involved 4-8 participants. Demographic information was collected using a demographics form. A semi-structured topic guide was used to facilitate the discussion. In total 38 participants took part in the study $(f=20, m=18)$. Participants identified themselves as heterosexual $(n=22)$, homosexual $(n=15)$ and "gay" $(n=1)$. Thematic analysis was used to analyse data and identify themes.
\end{abstract}

\section{Results}

Themes were grouped into three categories (see diagram). Theme 1 highlighted the diverse ways that relationships are understood and the fluid nature of partner types as they change across time and relationship development. Relationship types were also differentiated based on the prevalence of affective elements and emotions.

Theme 2 emphasised the contextual aspects that shape the way people form their social and sexual identities and also the way they talk about and perceive their sexual relationships. These aspects, in addition to previous experiences and access to sexual health services, are central in defining the ways people perceive STIs. Theme 3 underlined the use of the online communication in the formation of networks, the plurality of meanings and language used, the tailored online dating services meeting different user needs and finally the risk of deception.

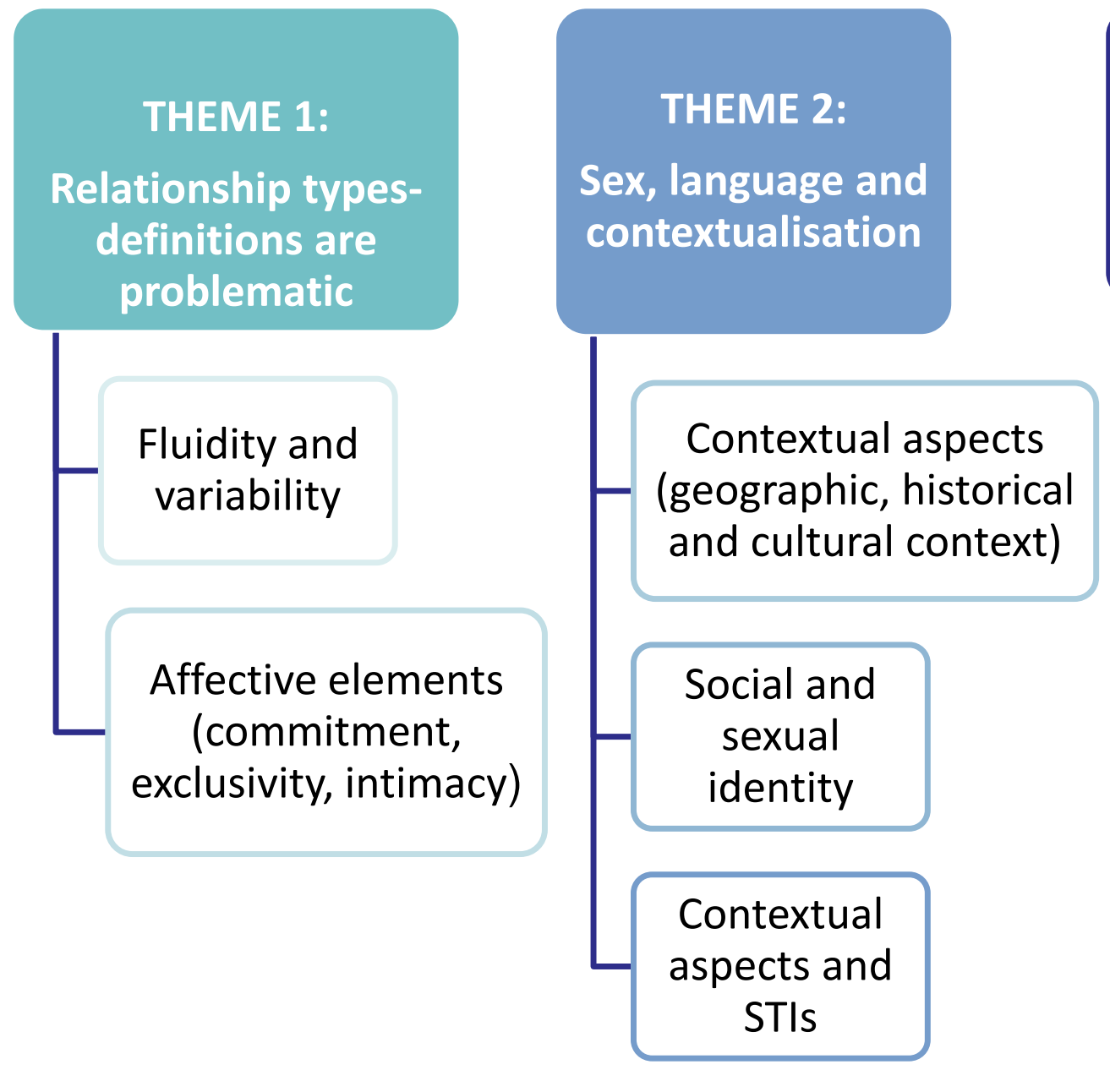

THEME 3:

Online dimension-a different world?

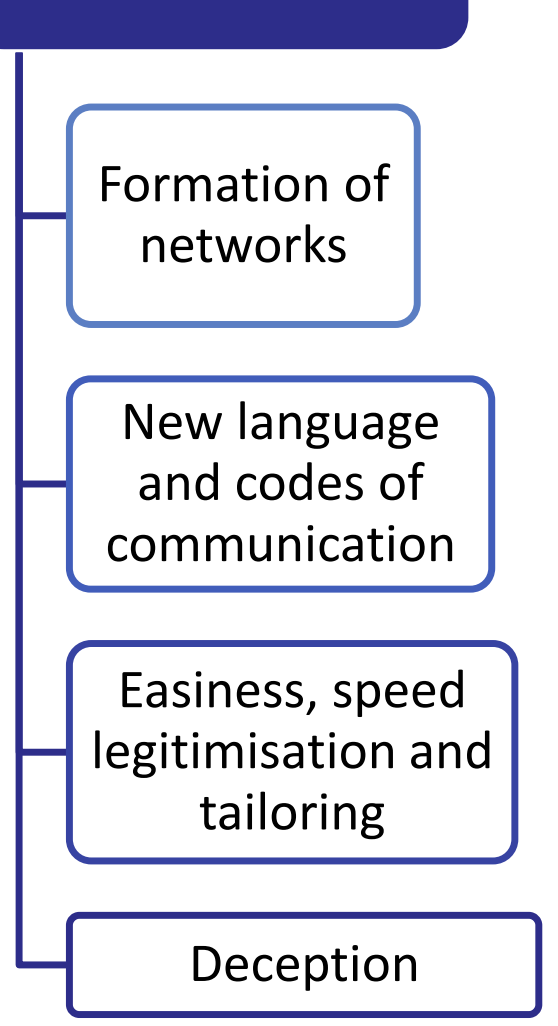

Conclusions

Social identities and social context 'shape' the way people perceive and talk about their sexual relationships. Patterns of STI transmission are related to social patterns, people's context and experiences, as well as previous experiences of accessing sexual health services. Prevention of STI transmission through partner notification could be improved by taking into consideration the contextual aspects of relationships in order to identify sexual networks and partner types that may yield the greatest public health benefit.

\section{References}

Adimora, A. \& Schoenbach, V. (2005) Social Context, Sexual Networks, and Racial Disparities in Rates of Sexually Transmitted Infections. The Journal of Infectious Diseases, 191, S115-22.

Harling G., Subramanian, SV., Bärnighausen, T., Kawachi, I. (2013) Socioeconomic disparities in Sexually Transmitted Infections among young adults in the United States: examining the interaction between income and race/ethnicity. Sexually Transmitted Diseases, 40(7): 575-581. doi:10.1097/OLQ.0b013e31829529cf.

Mercer, C.H., Jones K.G., Johnson A.M., Lewis R., Mitchell, K.R., Gravningen, K., Clifton, S., Tanton, C. Sonnenberg, P., Wellings, K., Cassell, J.A. \& Estcourt C.S. (2016) How can we objectively categorise partnership type? A novel classification of population survey data to inform epidemiological research and clinical practice. Sexually Transmitted Infections, 0, 1-8. doi:10.1136/sextrans-2016052646. 